\title{
Failure time in heterogeneous systems
}

\author{
Subhadeep Roy, ${ }^{1,2, *}$ Soumyajyoti Biswas $\odot,{ }^{3,4, \dagger}$ and Purusattam Ray ${ }^{5,6,+}$ \\ ${ }^{1}$ Earthquake Research Institute, University of Tokyo, 1-1-1 Yayoi, Bunkyo, 113-0032 Tokyo, Japan \\ ${ }^{2}$ PoreLab, Department of Physics, Norwegian University of Science and Technology, 7491 Trondheim, Norway \\ ${ }^{3}$ Max Planck Institute for Dynamics and Self-Organization, Am Fassberg 17, Göttingen, Germany \\ ${ }^{4}$ Department of Physics, SRM University - AP, Amaravati, Andhra Pradesh 522502, India \\ ${ }^{5}$ Institute of Mathematical Sciences, Taramani, Chennai 600113, India \\ ${ }^{6}$ Homi Bhabha National Institute, Training School Complex, Anushakti Nagar, Mumbai 400094, India
}

(Received 26 June 2019; published 24 October 2019)

\begin{abstract}
We show that the failure time $\tau_{f}$ in the fiber bundle model, taken as a prototype of heterogeneous materials, depends crucially on the strength of the disorder $\delta$ and the stress release range $R$ in the model. In the mean-field limit, the distribution of $\tau_{f}$ is log-normal. In this limit, the average failure time shows the variation $\tau_{f} \sim L^{\alpha(\delta)}$, where $L$ is the system size. The exponent $\alpha$ has a constant value above a critical disorder $\delta_{c}(=1 / 6)$, while it is an increasing function of $\delta$ in the region $\delta<\delta_{c}$. On the other hand, in the limit where the local stress concentration plays a crucial role, we observe the scaling $\tau_{f} \sim L^{\alpha(\delta)} \Phi\left(R / L^{1-\alpha(\delta)}\right)$, where $R$ is the stress release range. We find that the crossover length scale $R_{c}$, between the above two limiting cases, scales as $R_{c} \sim L^{1-\alpha(\delta)}$.
\end{abstract}

DOI: 10.1103/PhysRevResearch.1.033047

\section{INTRODUCTION}

In amorphous and heterogeneous materials, fracture processes involve a complex interplay of microcrack nucleation, coalescence, and growth of the cracks, leading to eventual failure of the materials [1]. In these materials, the process of fracture exhibits typically the following three stages: (i) initiation and formation of the microcracks at soft points of the sample, (ii) nucleation and coalescence of the microcracks, and (iii) the propagation of such microcracks [2]. Depending upon the disorder present in the material, however, the nucleating tendencies of the cracks can be inhibited [3]. In the limit of very high disorder, the nucleation process can be completely suppressed even up to the failure point, as was seen in the random fuse model [4]. For moderate disorder strengths, in spite of initial random failures, the final breakdown occurs through nucleation [5]. If, however, the timescale of nucleation is larger than that of the failure, the system might appear to demonstrate a pseudocriticality in these intermediate disorder strengths [5]. This pseudocriticality (or finite-size criticality) vanishes in the large-system-size limit, where the failure time overtakes the time required for the nucleation to set in. It was conjectured that the failure mode is essentially the outcome of the interplay between the disorder strength and the range $R$ up to which a local

\footnotetext{
*subhadeep.roy@ntnu.no

†soumyajyoti.b@srmap.edu.in

†ray@imsc.res.in
}

Published by the American Physical Society under the terms of the Creative Commons Attribution 4.0 International license. Further distribution of this work must maintain attribution to the author(s) and the published article's title, journal citation, and DOI. perturbation (failure) propagates within the system. A useful way to understand this interplay is the random fiber bundle model, where both of these parameters, the range of interaction and disorder strength, can be tuned independently [6,7]. A phase diagram depicting various modes of failures and the scaling criteria determining the different modes can be numerically evaluated [7].

In this work, we are interested in the question of the eventual failure time $\tau_{f}$ and its system-size scaling in the different fracture modes mentioned above. While the mode of failure is important information, the failure time is crucial from the point of view of effective engineering design and reliability [8-13] of structures. Many models have been proposed before to predict the failure time [12,14-18] of disordered materials. However, most models are concerned with the dependence of $\tau_{f}$ on applied load or on the macroscopic parameters like temperature and pressure, while treating the crack growth as an activation process $[13,16]$. The understanding of the failure time from the microscopic fracture dynamics, especially in the heterogeneous systems described above, has remained unclear $[19,20]$.

The time to failure of a mechanical system consisting of parallel membranes has been studied in the past [21,22]. These studies mostly have the order statistics of the failure time distribution for a single membrane. Damage evolution and time to failure have been investigated in a model where the damage formation is a stochastic event with the probability of failure at a point $i$ at time $t$ proportional to $\sigma_{i}^{\eta}(t)$, where $\sigma_{i}(t)$ is the local stress at $i$ at time $t$ [12]. The model predicts two regimes of failure: percolation like a failure for $\eta \leqslant 2$ and failure with precursory avalanches for $\eta>2$. A numerical study of the model on a two-dimensional triangular spring network model shows that for $\eta \leqslant 2$, the failure time is independent of the system size $L$, whereas for $\eta>2$, the failure time scales as $(\ln L)^{1-(\eta / 2)}[11]$. 
In the fiber bundle model [23] studied here, the number of failure modes is more than the two modes mentioned above. Here we look at the failure time and its scaling behaviors with system size $L$ for the different failure modes.

\section{DESCRIPTION OF THE MODEL}

The fiber bundle model [24,25] consists of a set of fibers attached between two parallel bars. One bar is kept fixed while the other one is pulled with external stress $\sigma$. Disorder is introduced into the model in the form of randomness in the failure strengths of the individual fibers. Here we consider a uniform probability distribution of the failure thresholds, centered around 0.5 and having a half-width $\delta$. When the applied stress crosses the failure strength of a fiber, that fiber breaks irreversibly. The stress on that broken fiber is then redistributed among the remaining intact fibers. There are broadly two schemes to redistribute the stress: the equal load sharing (ELS) scheme and the local load sharing (LLS) scheme. In the ELS scheme, the stress is redistributed uniformly among all other surviving fibers $[25,26]$. In the LLS scheme, only the nearest surviving neighbors of the broken fiber carry the extra load [27-32]. There are several intermediate load sharing schemes that interpolate between these two limiting cases, such as a power-law load sharing [33] or load sharing within a fixed range $R[6,7]$. After the redistribution, there might be further breaking events, initiating an avalanche. When the system eventually comes to a stable configuration, the applied stress is increased again to break the next weakest fiber and the process continues until all the fibers break.

In this study, however, we are solely interested in the failure time $\tau_{f}$. In measuring that, first we find the critical load $\sigma_{c}$, which a fiber bundle can just withstand. For every configuration, the system is loaded marginally above this critical load such that it eventually breaks down without any further increase in the load. The number of redistribution steps taken by the system, from the initial application of the load to the final breakdown, is noted as the failure time $\tau_{f}$.

\section{ANALYTICAL APPROACH: MEAN-FIELD LIMIT}

In the mean-field limit, the effect of the disorder strength on the time evolution of $U(t, \sigma, \delta)$ can be approximately determined; $U(t, \sigma, \delta)$ is the the fraction of surviving fibers at time $t$, at stress $\sigma$, and for the half-width $\delta$ of the threshold distribution. Then $U(t, \sigma, \delta)$ satisfies the recursion relation [34]

$$
U(t+1, \sigma, \delta)=\frac{1}{2 \delta}\left((c+\delta)-\frac{\sigma}{U(t, \sigma, \delta)}\right),
$$

where $c$ is the mean of threshold stress distribution; $c$ and $\delta$ have dimensions similar to the stress. At the critical point, $\sigma$ will be replaced by the critical stress $\sigma_{c}$. A recent study [35] shows that there exists a critical point at $\delta_{c}$ around which the mode of failure changes from brittle (abrupt) to quasibrittle (nonabrupt) with appropriate critical scalings. In the quasibrittle region $\left(\delta \geqslant \delta_{c}\right)$ we already have an expression for $\sigma_{c}[35]$ :

$$
\sigma_{c}=\frac{\delta}{2}\left(1+\frac{c-\delta}{2 \delta}\right)^{2}
$$

Then, at the critical point in the quasibrittle regime we get, from Eq. (1),

$$
U\left(t, \sigma_{c}, \delta\right)-U_{c}=\left(\frac{1}{2}+\frac{1}{4 \delta}\right) t^{-1},
$$

where $U_{c}$ is the fraction of unbroken fibers at critical stress. At $\delta=0.5, U_{c}=0.5$ and the above result matches with the behavior $U\left(t, \sigma_{c}, \delta=0.5\right)-U_{c} \sim 1 / t$ observed before [34]. Also, this behavior is independent of $\delta$, as long as $\delta \geqslant \delta_{c}$. In the brittle region $\left(\delta<\delta_{c}\right)$, the picture is quite different. The disorder distribution is so narrow that the system fails as soon as the weakest fiber in the system breaks. However, due to sample to sample fluctuation, the weakest fiber in a system does not necessarily have the lower bound of the threshold distribution as its failure strength. This difference also depends on the system size. In this region we can write $\sigma_{c}=\sigma_{l}+\epsilon$, where $\sigma_{l}$ is the lower bound of the threshold distribution and is given by $c-\delta$. In addition, $\epsilon$ is the term that takes care of the finite-size effect reflected through deviation of the strength of the weakest fiber from the lower bound of the threshold distribution. As we go to higher system sizes, the magnitude of $\epsilon$ decreases. The recursion relation in this case takes the form

$U\left(t+1, \sigma_{c}, \delta\right)=1+A\left(1-\frac{1}{U\left(t, \sigma_{c}, \delta\right)}\right)-\frac{\epsilon}{2 \delta U\left(t, \sigma_{c}, \delta\right)}$,

where $A=\frac{\sigma_{l}}{2 \delta}$. As $U\left(0, \sigma_{c}, \delta\right)=1$ its easy to see that $U\left(1, \sigma_{c}, \delta\right)=1-\frac{\epsilon}{2 \delta}$. Repeating this recursively, we get

$$
U\left(t, \sigma_{c}, \delta\right)=1-\frac{\epsilon}{2 \delta-\epsilon} \frac{1-A^{t}}{1-A},
$$

where the higher-order terms in $\epsilon$ are neglected. Using the expression of $A$, we get

$$
U\left(t, \sigma_{c}, \delta\right)=1-\frac{4 \delta \epsilon}{(2 \delta-\epsilon)(6 \delta-1)}\left[1-\left(\frac{1-2 \delta}{4 \delta}\right)^{t}\right] .
$$

In Fig. 1 we have compared the analytical expression of $U\left(t, \sigma_{c}\right)$ with the numerical behavior generated for system sizes ranging from $10^{4}$ to $4 \times 10^{5}$ and with $10^{4}$ configurations. Here $\delta$ is kept constant at 0.14 . The choice of $\delta$ is rather free as long as the model remains in the brittle region $\left(\delta<\delta_{c}\right)$. We treat $\epsilon$ as a fitting parameter here, expecting it to decrease as $L$ is increased, making the difference between $\sigma_{c}$ and $\sigma_{l}$ smaller. The points correspond to the numerical results where the lines are drawn using Eq. (6) for certain $\delta$ and $\epsilon$ values, as indicated. The envelope of the curve in Fig. 1 increases as we go to higher system sizes, suggesting an increment in failure time when the system size is increased. We also observe that as the system size is increased, $\epsilon$ decreases as $\epsilon \sim 1 / L$. Such behavior is consistent with the weakest link of chain approach [36]. As higher-order terms are neglected, the analytical result does not tally with the numerical findings at long times. For this reason Eq. (6) cannot be used to predict the size dependence of $\tau_{f}$. For that we need to resort to numerical simulations. 

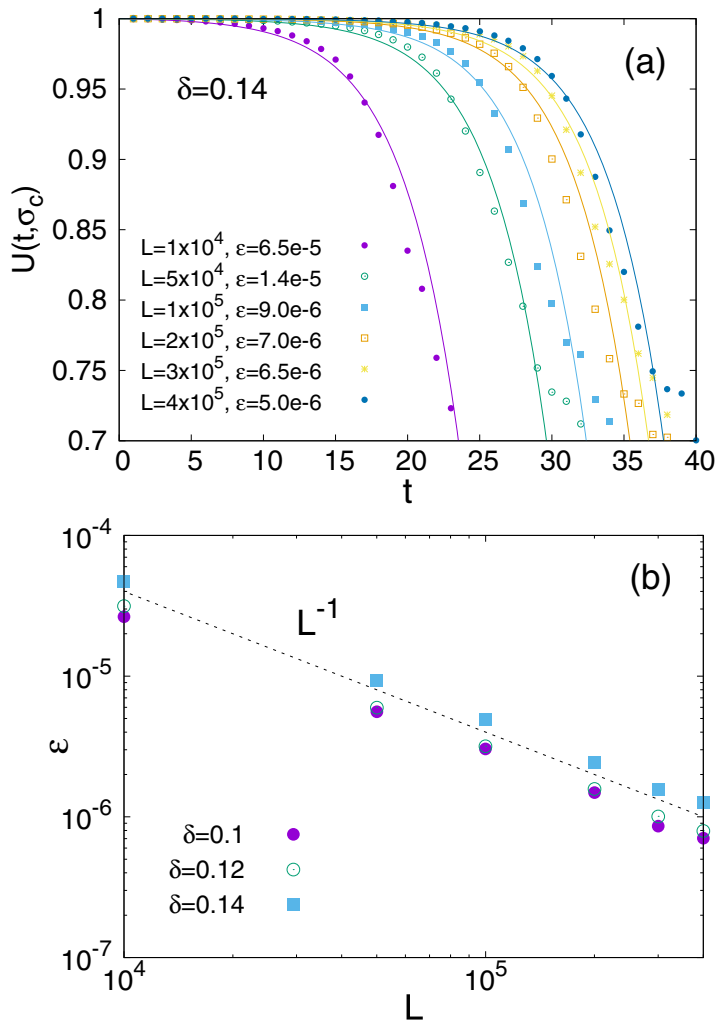

FIG. 1. (a) Study of $U\left(t, \sigma_{c}\right)$ with increasing time steps for system sizes ranging between $10^{4}$ and $4 \times 10^{5}$. Disorder is kept fixed at $\delta=0.14$. The points show the numerical results, while the lines follow the analytical results given by Eq. (6). The value of $\epsilon$ is adjusted depending on $\delta$. The envelope of the curve increases and $\epsilon$ decreases as we go to higher system sizes. (b) Plot of $\epsilon$ decreasing with system size $L$ as $\epsilon \sim 1 / L$. Such scale-free behavior is observed to be independent of the strength of the disorder $\delta$.

\section{NUMERICAL RESULTS}

With the analytical approach outlined above, we could not find the correct system-size scaling of the failure time, nor could we go beyond the mean-field limit. In this section, we determine the system-size scaling of the failure time $\tau_{f}$ in different failure modes (controlled by changing $\delta$ ) for both the mean-field and finite-range interaction $R$.

The failure time $\tau_{f}$ is the envelope of the $U\left(t, \sigma_{c}, \delta\right)$ vs $t$ curve. We have determined it numerically for different $\delta$ and $R$. Numerically, $\tau_{f}$ is estimated as the number of redistributing steps that the fiber bundle goes through under a loading slightly above the critical load, until its global failure. We have followed the definition of the interaction range $R$ as the number of surviving fibers on both sides of the broken one among which the extra stress of the broken fiber is uniformly redistributed [6]. Numerical simulations are carried out with a bundle of size ranging between $10^{3}$ and $4 \times 10^{5}$ and realizations between $10^{2}$ and $10^{5}$.

\section{A. Failure dynamics in the mean-field limit}

Here we have shown the evolution of the surviving fraction of fibers $U(t)$ with time in the mean-field limit, when the system is loaded with stress slightly higher than the critical

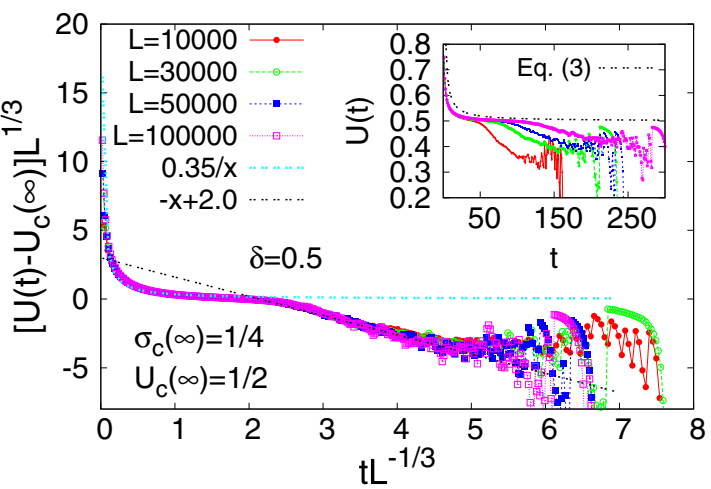

FIG. 2. Time evolution of the unbroken fraction for $\delta=0.5$ while a critical stress $\sigma_{c}(\infty)$ is applied on it in the mean-field limit. The main figure and the inset show the scaled and unscaled results, respectively.

value. Under such a condition, the bundle will break in a single avalanche and over time (or load redistribution steps) $\tau_{f}$, which is the failure time of the bundle. We discuss results for both the quasibrittle $\left(\delta>\delta_{c}\right)$ and the brittle (abrupt failure) $\left(\delta<\delta_{c}\right)$ regions.

\section{Quasibrittle region}

We set $\delta=0.5$. For this strength of disorder, the system is deep in the quasibrittle region (since $\delta_{c}=1 / 6$ ) and $\sigma_{c}(\infty)$ and $U_{c}(\infty)$ are known exactly to be 0.25 and 0.5 , respectively, in the thermodynamic limit $(L \rightarrow \infty)$.

The inset of Fig. 2 shows the unscaled behavior of $U(t)$ where it starts from 1 and subsequently decays rapidly with increasing time. The results are shown for four different system sizes ranging between $10^{4}$ and $10^{5}$. The blue dotted line compares the numerical results with the analytical findings in the preceding section and it shows the similarities in the low- $t$ limit.

The scaling behavior of $U(t)$ is

$$
[U(t)-U(\infty)] L^{\alpha} \sim \Psi_{1}\left(t L^{-\alpha}\right),
$$

where $\alpha=1 / 3$. The exponent $\alpha$ remains the same throughout the region $\delta>\delta_{c}(=1 / 6)$. From the scaling equation (7), we can study the behavior in two different limits.

(i) When $t$ is small, we observe that the function $\Psi_{1}(x)$ behaves as $1 / x$. In the limit $t \ll L^{1 / 3}$ we get

$$
U(t)-U(\infty) \sim \frac{1}{t} .
$$

(ii) For large $t$, where $t \gg L^{1 / 3}$, we observe $\Psi_{1}(x) \sim-x$ and in that limit $U(t)$ behaves as

$$
U(t)-U(\infty) \sim-\frac{t}{L^{2 / 3}} .
$$

The failure time $\tau_{f}$ can be obtained by setting Eq. (7) to a constant value as there will be no evolution of the unbroken fraction with time. Setting the right-hand side of the same equation to a constant value, we get

$$
\left.t L^{-\alpha}\right|_{t=\tau_{f}}=\text { const }
$$



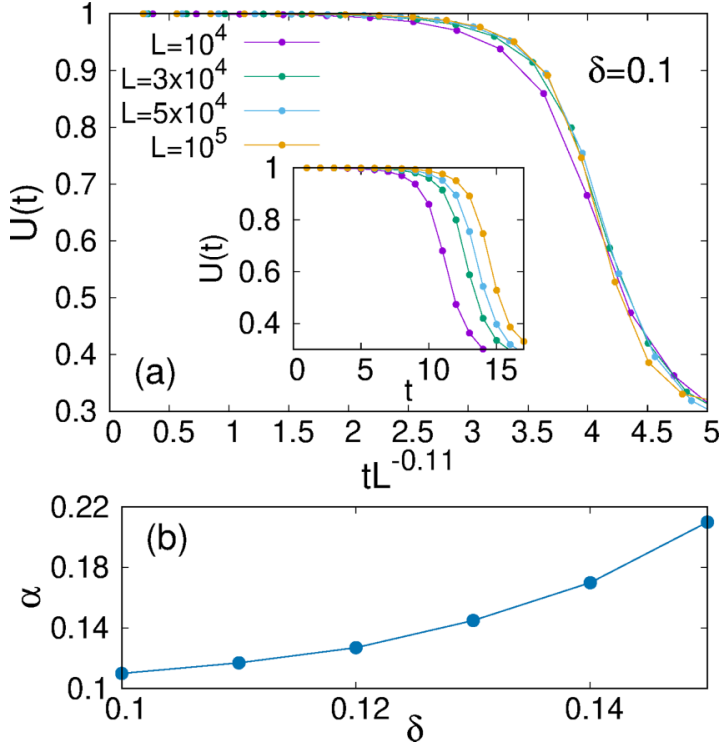

FIG. 3. (a) Time evolution of the fraction of unbroken $U(t)$ in the brittle region $\delta<\delta_{c}$. We have observed the scaling $U(t) \sim$ $t L^{-\alpha}$. (b) Unlike the quasibrittle region, the exponent $\alpha$ increases monotonically with the strength of disorder.

or

$$
\tau_{f} \sim L^{1 / 3}
$$

The results of Eqs. (8) and (10) are consistent with what was observed in the fiber bundle model in the mean-field limit [37].

\section{Brittle region}

In the brittle region $\left(\delta<\delta_{c}\right)$, due to the abrupt nature of the failure, $U(t)$ decreases very fast with time (see Fig. 3). The critical stress in this region coincides with the threshold value of the weakest fiber.

Simulations have been carried out for system sizes ranging between $10^{4}$ and $10^{5}$ with disorder strength $\delta=0.1$. Here we observe the scaling with system size

$$
U(t) \sim \Psi_{2}\left(t L^{-\alpha}\right),
$$

where the exponent $\alpha$ increases monotonically with strength of disorder $\delta$. The scaling remains the same throughout the region $\delta<\delta_{c}(=1 / 6)$. As a failure criterion we can set the right-hand side of Eq. (11) to a constant value. The scaling of the failure time with size of the system can be expressed as

$$
\left.t L^{-\alpha(\delta)}\right|_{t=\tau_{f}}=\text { const }
$$

or

$$
\tau_{f} \sim L^{\alpha(\delta)}
$$

The scaling of $\tau_{f}$ with system size $L$ at different disorder strength values will be revisited numerically later in this paper.

Figure 4 shows the system-size effect of the average failure time $\tau_{f}$ at different disorder values. Here $\tau_{f} \sim L^{\alpha}$ for all $\delta$, with $\alpha$ the exponent of the power law. Above $\delta_{c}, \alpha$ remains constant, independent of $\delta$. In vanishingly small disorder, the model is bound to fail in the redistributing step independent of

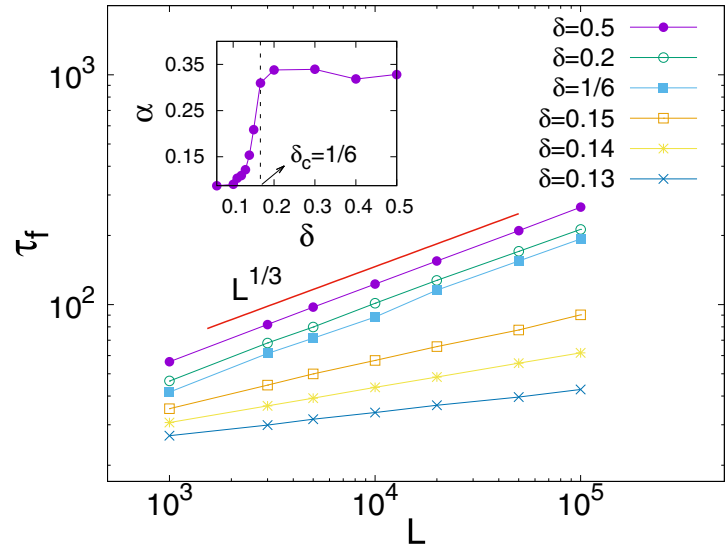

FIG. 4. System-size effect of the maximum average relaxation time $\tau_{f}$ at different $\delta$ value. Here $\tau_{f}$ shows a scale-free behavior with system size $L: \tau_{f} \sim L^{\alpha}$. The value of the exponent $\alpha$ remains constant at $1 / 3$ for $\delta>\delta_{c}$, while it keeps decreasing below $\delta_{c}$ and the system-size effect of $\tau_{f}$ gradually vanishes. The inset shows the variation of the scaling exponent $\alpha$ with a varying disorder value. For $\delta>\delta_{c}, \alpha$ saturates at a value $1 / 3$. Below $\delta_{c}$ the exponent value decreases as we go to lower $\delta$ values.

system size (essentially in one single step, as all the fibers will have the same failure threshold). As the model approaches this vanishingly small disorder limit $(\delta \rightarrow 0)$, the exponent
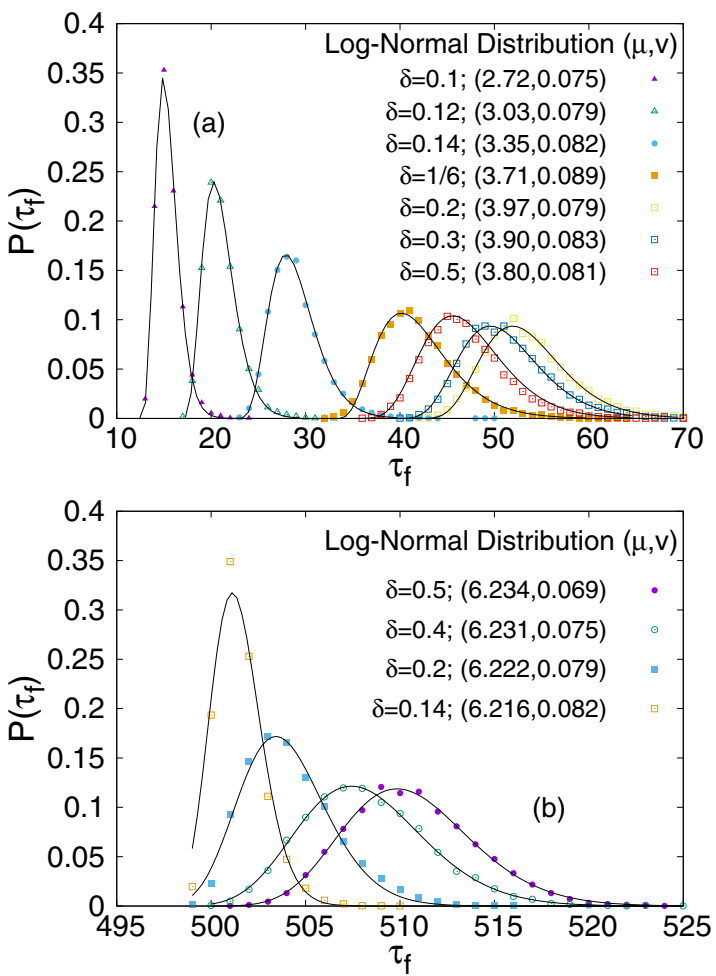

FIG. 5. Distribution of the failure time for different $\delta$ fitted for (a) the mean-field limit $R>R_{c}$ and (b) extreme local stress concentration limit $R=1\left(<R_{c}\right)$, with $R_{c}$ the critical value of the stress release range around which a transition from LLS to the mean field was observed (see Ref. [6] as well as the next section for details). The numerical results suggest a nice fit with a log-normal distribution with mean $\mu$ and variance $v$. 

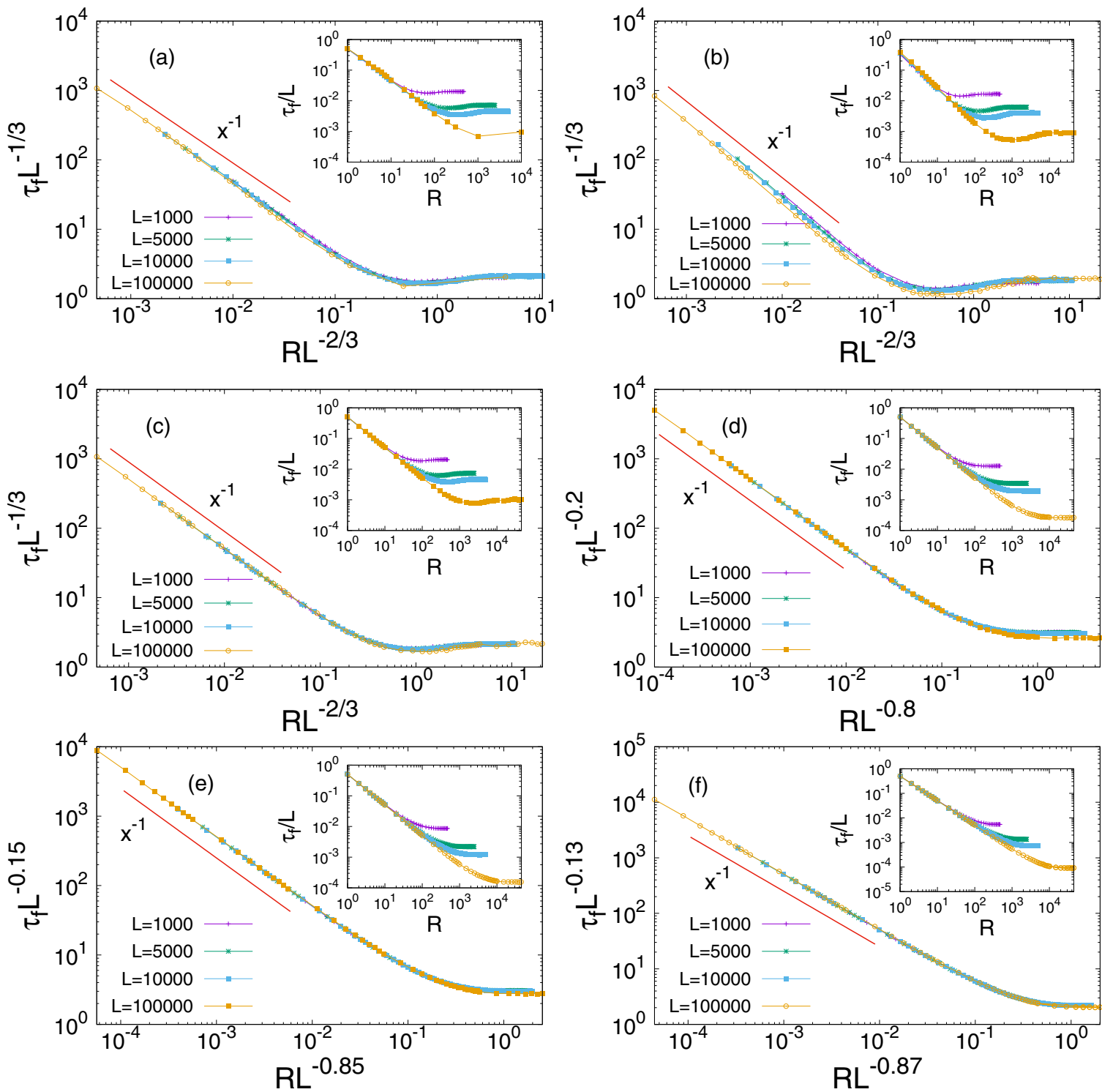

FIG. 6. Scaling of the failure time $\tau_{f}$ with system sizes $10^{3}, 5 \times 10^{3}, 10^{4}$, and $10^{5}$ for disorder (a) $\delta=0.5$, (b) 0.3 , (c) $\delta=1 / 6$, (d) $\delta=0.13$, (e) $\delta=0.1$, and (f) $\delta=0.06$. The inset shows the unscaled behavior of $\tau_{f}$. The scaling behavior shows $\tau_{f} \sim L^{\alpha(\delta)} \Phi\left(R / L^{1-\alpha(\delta)}\right)$. The value of $\alpha$ remains the same (1/3) in the region $\delta>\delta_{c}$. For $\delta<\delta_{c}$, the value of this exponent $\alpha$ behaves similarly to what was shown in Fig. 3(b) and the inset in Fig. 4.

$\alpha$ decreases. The failure time $\tau_{f}$ satisfies the scaling behavior

$$
\tau_{f} \sim \begin{cases}L^{1 / 3} & \text { for } \delta>\delta_{c} \\ L^{\alpha(\delta)} & \text { for } \delta<\delta_{c}\end{cases}
$$

where $\alpha(\delta)$ is an increasing function of $\delta$.

In the mean-field limit, the relaxation time at critical stress has been found before to diverge as $L^{1 / 3}$ [37]. At the same time, the relaxation time diverges as we approach $\delta_{c}$ as $L^{1 / 3}$ [35]. The present study shows a similar scaling behavior in the limit $\delta<\delta_{c}$ as well with a disorder-dependent exponent $\alpha$.

Distribution of failure time. Figure 5 shows the distribution of failure times $\tau_{f}$ at different $\delta$ values for system-size $L=$ $10^{3}$ and $10^{5}$ realizations. The distribution of $\tau_{f}$ fits satisfactorily with the log-normal distribution. The behavior of the fitting is discussed below in detail.
The log-normal distribution that we use for the fitting is given by

$$
P\left(\tau_{f}\right)=\frac{1}{\tau_{f} v \sqrt{2 \pi}} \exp \left[-\frac{\left(\ln \tau_{f}-\mu\right)^{2}}{2 v^{2}}\right] .
$$

Here $\mu$ and $v$ are, respectively, the mean and variance of the distribution. The fitting of failure time for different $\delta$ values is shown explicitly in Fig. 5 for both $R>R_{c}$ (mean field) and $R<R_{c}$ ( $R=1$ to be specific), where $R_{c}$ is the critical value of the stress release range around which the model goes from LLS to the mean-field limit. A more detailed discussion of $R_{c}$ can be found in the next section. The results are summarized below.

(i) In the mean-field limit $\left(R>R_{c}\right)$, the distribution for $\tau_{f}$ is log-normal irrespective of the strength of disorder. 
(ii) With local stress concentration $\left(R<R_{c}\right)$, the distribution is log-normal only for $\delta>\delta_{c}$. For $\delta<\delta_{c}$, the distribution is a $\delta$ function at $L / 2 R$ (which is 500 in this case as $R=1$ and $L=10^{3}$ ). In this region $\delta_{c}$ changes with system size $L$, as discussed in detail in a recent paper [38].

The two factors that mainly control the failure time are how close the threshold values of the fibers are and what the applied critical stress value is. The interplay between these two factors determines the average position of the failure time distribution. In the mean-field limit, the average failure time reaches its highest value at an intermediate strength of disorder where the applied stress is close to the threshold of the weakest link and at the same time there is a moderate fluctuation among the strength values from fiber to fiber. At higher disorder, the critical stress is well inside the distribution and breaks a large part of the bundle at the beginning, which in turn leads to high redistribution stress. On the other hand, at low disorder, the threshold values are very close to each other, increasing the probability of multiple fiber breaking at a single time step. Both of these cases eventually lead to a shorter failure time [see Fig. 5(a)]. Such behavior is not observed with local stress concentration as the long-range interaction is absent here and a weak fiber will still survive (after the redistribution) at a time $t$ if it is not a neighbor of the broken fiber. The average of the distribution moves to a higher value with increasing strength of disorder in this limit [see Fig. 5(b)].

In addition to the log-normal distribution, we have fitted our numerical results with the Weibull distribution. The goodness of the fitting is determined from the $\chi^{2}$ deviation and the fitting with the log-normal distribution is observed to be more reliable (For further details, see the Appendix).

\section{B. Failure dynamics with local stress concentration}

Finally, we arrive at the point where we can study the model with local stress concentration. For this purpose, we consider that the stress of a broken fiber is redistributed among $R$ surviving nearest neighbors. For the one-dimensional fiber bundle model, $R=1$ corresponds to the LLS limit, while $R=L / 2$ leads to the mean-field limit of the model. Since the perturbation due to a broken fiber cannot reach more than $R$ nearest surviving neighbors, the model will produce a failure time $\tau_{f}=L / R$ even if all the threshold values are the same. Instead, we can observe here how the spatial correlation is affected by the strength of disorder, while the stress release range $R$ is continuously varied. A recent study shows that the failure process is nucleating or spatially correlated if the stress redistribution is slower than a critical range $R_{c}\left(\sim L^{2 / 3}\right)$ [6]. The observation in that study was carried out while the threshold values were chosen from a uniform distribution in $(0,1)$. In this section, we explore the behavior of such an $R_{c}$ in one dimension when the strength of disorder is varied within a wide range, making the failure process both brittle and quasibrittle. The study of the scaling of $R_{c}$ in low disorder values will tell how the rupture pattern is modified when the strength of the disorder is low.

Figure 6 shows the scaling of $\tau_{f}$ with the range $R$ for different system sizes ranging from $10^{3}$ to $10^{5}$ and for different disorder strengths. We observe the scaling of $\tau_{f}$ with $R$ and system size $L$ for all $\delta$ values,

$$
\tau_{f} \sim L^{\alpha(\delta)} \Phi\left(\frac{R}{L^{1-\alpha(\delta)}}\right)
$$

where the scaling exponent $\alpha$ is a function of $\delta$ and behaves in the same way observed in Figs. 3 or 4 . The inset of Fig. 6 shows the unscaled variation of $\tau_{f} / L$ with $R$ at different system sizes. The above scaling is consistent with the behavior observed earlier [6]. The scaling function $\Phi(x)$ behaves as $1 / x$ below the critical range $R_{c}$. This leads to $\tau_{f}=L / R$ in the regions $R<R_{c}$. On the other hand, beyond $R_{c}, \alpha=1 / 3$ and $\Phi(x)$ is just a constant. This gives us $\tau_{f} \sim L^{1 / 3}$ beyond $R_{c}$. These two $\tau_{f}$ values should agree with each other at $R_{c}$. This condition offers us the scaling of $R_{c}$ with the system size $L: R_{c} \sim L^{1-\alpha(\delta)}$. This suggests that, in the brittle region, as the strength of disorder is decreased, we have to go to a higher range of stress redistribution to achieve spatially uncorrelated rupture events.

\section{DISCUSSION AND CONCLUSION}

We have studied the variation of failure time with disorder strength, range of interaction, and system sizes in the fiber bundle model. The system was first marginally overloaded and the number of stress redistributions required for the entire system to break was taken as the failure time. In this way, even for the brittle region, there is a finite failure time. The system-size scaling and the distributions of the failure times show intriguing behavior for different disorder strengths and interaction ranges. These are summarized in Table I. In particular, for a very local interaction range $\left(R<R_{c}\right)$, the failure time is inversely proportional to $R$. This is the region of damage nucleation, which eventually turns into a diffused

TABLE I. Behavior of the failure time distribution and size effect of $\tau_{f}$ in all four regions: the brittle mean field $\left(R>R_{c}\right.$ and $\left.\delta<\delta_{c}\right)$, the quasibrittle mean field $\left(R>R_{c}\right.$ and $\left.\delta>\delta_{c}\right)$, the brittle mean field with local stress concentration $\left(R<R_{c}\right.$ and $\left.\delta<\delta_{c}\right)$, and the quasibrittle mean field with local stress concentration $\left(R<R_{c}\right.$ and $\left.\delta>\delta_{c}\right)$. The boundaries of these regions have been observed in recent papers. Here $\delta_{c}$ demarcates the brittle and quasibrittle regions (see Ref. [35]), while $R_{c}$ separates the mean-field limit from local load sharing (see Ref. [6]). The scaling of $\delta_{c}$ with system size in the region $R<R_{c}$ is discussed in Ref. [38].

\begin{tabular}{lcc}
\hline \hline Range & $\delta>\delta_{c}$ & $\delta<\delta_{c}$ \\
\hline$R>R_{c}$ & distribution of $\tau_{f}:$ log-normal & distribution of $\tau_{f}:$ log-normal \\
$R>R_{c}$ & $\tau_{f} \sim L^{1 / 3}$ & $\tau_{f} \sim L^{\alpha(\delta)}$ where $\alpha$ increases with $\delta$ \\
$R<R_{c}$ & distribution of $\tau_{f}:$ log-normal & distribution of $\tau_{f}: \delta$ function at $L / 2 R$ \\
$R<R_{c}$ & $\tau_{f} \sim L / R$ & $\tau_{f} \sim L / R$ \\
\hline \hline
\end{tabular}


damage and avalanche dynamics when $R$ is sufficiently large. Consequently, the failure time distribution turns from a $\delta$ function to a log-normal distribution. The log-normal and similar (e.g., Weibull) distributions have been found before for failure time distributions in the case of creep rupture. Creep failure has been studied [39-43] extensively both experimentally and theoretically from the point of view of statistical mechanics. In Refs. [40-42], a log-normal and a Weibull distribution were observed for $1 / 2 \mathrm{Cr} 1 / 2 \mathrm{Mo} 1 / 4 \mathrm{~V}$ steel (1Cr1Mo1/4V steel) and Ti-6246 alloy, respectively. The same Weibull distribution was found for STS304 stainless steel [43]. Generally, in creep rupture, the systems are loaded below their critical point and the thermal (or other external) noise takes the systems over the failure threshold. In our case, however, there is no external noise. It is the external loading, which is controlled to be slightly above the critical point, that causes the eventual failure of the system. Notwithstanding this difference in the mechanism for the failure initiations, the behavior of the failure time distributions we find here is very similar to that found for creep rupture experiments. Our results are universal in the sense that the scalings are valid for different forms of failure threshold distributions, i.e., a uniform distribution (shown here) and a power-law distribution with exponent -1 .

In conclusion, we have found that microscopic parameters such as the strength of the disorder and the range of stress redistribution have a profound effect on the failure time of a disordered sample. We have found the scaling relation of the failure time for the full parameter space of the system size, the strength of disorder, and the stress release range. A universal scaling exponent was observed beyond the critical strength of the disorder value. As a result of the extreme statistics of the fracture dynamics, the distribution of failure time was found to be log-normal at any strength of disorder, unless the range of interaction was very low.

\section{APPENDIX}

Figure 7 shows the distribution of failure times $\tau_{f}$ at different $\delta$ values for system size $L=10^{3}$ and $10^{5}$ realizations. The distribution of $\tau_{f}$ has been fitted satisfactorily with two different distributions: Weibull and log-normal. The behavior of the fitting is discussed below in detail.

The Weibull distribution that we have adopted is given by

$$
P\left(\tau_{f}\right)=\left(\frac{k}{\lambda}\right)\left(\frac{\tau_{f}-\gamma}{\lambda}\right)^{k-1} \exp \left[-\left(\frac{\tau_{f}-\gamma}{\lambda}\right)^{k}\right],
$$

where $k, \lambda$, and $\gamma$ are, respectively, the shape, scale, and position parameters of the distribution. In contrast, the lognormal distribution is given by

$$
P\left(\tau_{f}\right)=\frac{1}{\tau_{f} v \sqrt{2 \pi}} \exp \left[-\frac{\left(\ln \tau_{f}-\mu\right)^{2}}{2 v^{2}}\right] .
$$

Here $\mu$ and $v$ are, respectively, the mean and variance of the distribution. The fitting of the failure time with these two distributions is shown explicitly in Fig. 7. The goodness of fit has been checked from the study of reduced $\chi^{2}$ values.
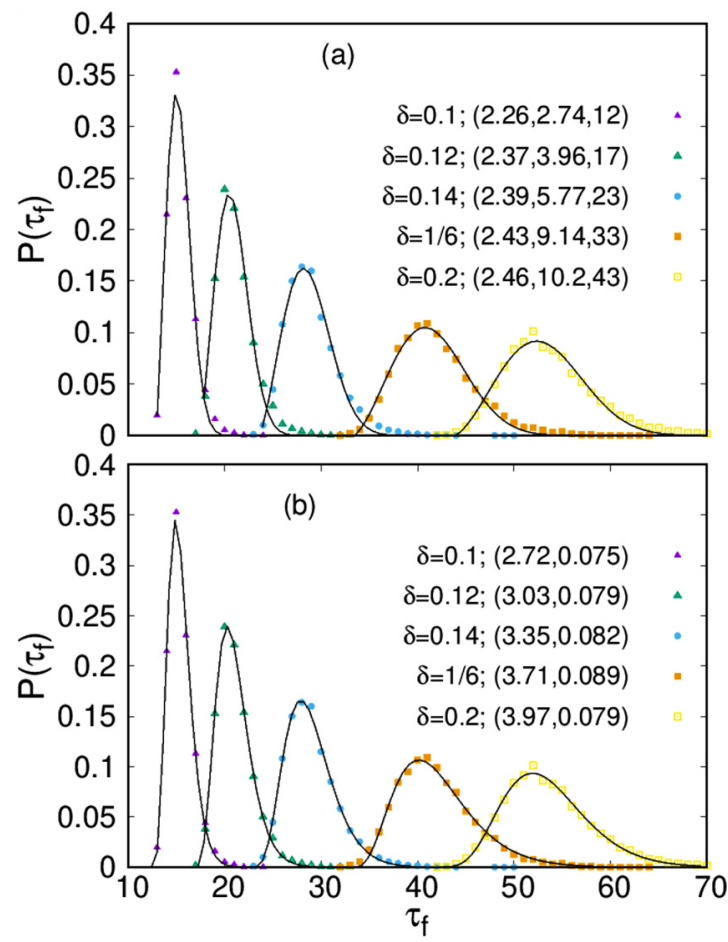

FIG. 7. Distribution of the failure time for different $\delta$ fitted with (a) the three-parameter Weibull distribution and (b) the lognormal distribution. The numerical results are fitted with different parameters such as the shape, scale, and position parameter for the Weibull distribution and the mean and variance for the log-normal distribution.

Reduced $\chi^{2}$ (denoted by $\chi_{r}^{2}$ ) is basically defined as the $\chi^{2}$ value per degree of freedom $v$ (equal to the number of observations minus the number of fitted parameters) defined as

$$
\chi_{r}^{2}=\frac{1}{v} \sum \frac{\left(O_{i}-E_{i}\right)^{2}}{E_{i}},
$$

where $O_{i}$ and $E_{i}$ are observed and expected data, respectively. In Table II we provide a comparison of reduced $\chi_{r}^{2}$ for both distributions. This comparison shows that, for both cases, $\chi_{r}^{2}$ has a lower value and the fit of the numerical data is satisfactory. The log-normal distribution can be claimed to be fitted more appropriately as $\chi_{r}^{2}$ is an order lower than that of the Weibull distribution.

TABLE II. Comparison of the reduced $\chi_{r}^{2}$ deviation for lognormal and Weibull distributions as the distribution of the failure time.

\begin{tabular}{lcc}
\hline \hline & \multicolumn{2}{c}{$\chi_{r}^{2}$} \\
\cline { 2 - 3 }$\delta$ & Weibull distribution & log-normal distribution \\
\hline 0.1 & $1.63 \times 10^{-4}$ & $3.5 \times 10^{-5}$ \\
0.12 & $8.42 \times 10^{-5}$ & $7.25 \times 10^{-6}$ \\
0.14 & $3.66 \times 10^{-5}$ & $8.59 \times 10^{-6}$ \\
$1 / 6$ & $1.21 \times 10^{-5}$ & $2.45 \times 10^{-6}$ \\
0.2 & $1.45 \times 10^{-5}$ & $4.41 \times 10^{-6}$ \\
\hline
\end{tabular}


[1] L. B. Freund, Dynamic Fracture Mechanics, Cambridge Monographs on Mechanics (Cambridge University Press, Cambridge, 1998).

[2] T. Shioya, Y. Koga, K. Fujimoto, and R. Ishida, J. Phys. Colloques 49, C3-253 (1988).

[3] N. Shahidzadeh-Bonn, P. Vie, X. Chateau, J. N. Roux, and D. Bonn, Phys. Rev. Lett. 95, 175501 (2005).

[4] A. A. Moreira, C. L. N. Oliveira, A. Hansen, N. A. M. Araujo, H. J. Herrmann, and J. S. Andrade, Phys. Rev. Lett. 109, 255701 (2012).

[5] A. Shekhawat, S. Zapperi, and J. P. Sethna, Phys. Rev. Lett. 110, 185505 (2013).

[6] S. Biswas, S. Roy, and P. Ray, Phys. Rev. E 91, 050105(R) (2015).

[7] S. Roy, S. Biswas, and P. Ray, Phys. Rev. E 96, 063003 (2017).

[8] L. Pauchard and J. Meunier, Phys. Rev. Lett. 70, 3565 (1993).

[9] D. Bonn, H. Kellay, M. Prochnow, K. Ben-Djemiaa, and J. Meunier, Science 280, 265 (1998).

[10] A. Lucantonio, G. Noselli, X. Trepat, A. DeSimone, and M. Arroyo, Phys. Rev. Lett. 115, 188105 (2015).

[11] W. A. Curtin, M. Pamel, and H. Scher, Phys. Rev. B 55, 12051 (1997).

[12] W. A. Curtin and H. Scher, Phys. Rev. B 55, 12038 (1997).

[13] A. Guarino, S. Ciliberto, A. Garcimartın, M. Zei, and R. Scorretti, Eur. Phys. J. B 26, 141 (2002).

[14] L. Golubović and S. Feng, Phys. Rev. A 43, 5223 (1991).

[15] Y. Pomeau, C. R. Acad. Sci. Paris, Série II 314, 553 (1992).

[16] A. Rabinovitch, M. Friedman, and D. Bahat, Europhys. Lett. 67, 969 (2004).

[17] A. Politi, S. Ciliberto, and R. Scorretti, Phys. Rev. E 66, 026107 (2002).

[18] H. M. Taylor, Adv. Appl. Probab. 11, 527 (1979).

[19] G. E. Dieter, Mechanical Metallurgy (McGraw-Hill, New York, 1986).

[20] B. Lawn, Fracture of Brittle Solids, 2nd ed. (Cambridge University Press, Cambridge, 1993).

[21] S. L. Phoenix, SIAM J. Appl. Mech. 34, 227 (1978).
[22] Z. P. Bazant and J. L. Lee, Eng. Failure Anal. 16, 2521 (2009).

[23] A. Hansen, P. C. Hemmer, and S. Pradhan, The Fiber Bundle Model: Modeling Failure in Materials (Wiley-VCH, Berlin, 2015).

[24] S. Pradhan, A. Hansen, and B. K. Chakrabarti, Rev. Mod. Phys. 82, 499 (2010).

[25] F. T. Pierce, J. Text. Inst. Trans. 17, 355 (1926).

[26] H. E. Daniels, Proc. R. Soc. London Ser. A 183, 405 (1945).

[27] S. L. Phoenix, Adv. Appl. Probab. 11, 153 (1979).

[28] R. L. Smith and S. L. Phoenix, J. Appl. Mech. 48, 75 (1981).

[29] W. I. Newman and S. L. Phoenix, Phys. Rev. E 63, 021507 (2001).

[30] D. G. Harlow and S. L. Phoenix, J. Compos. Mater. 12, 314 (1978).

[31] D. G. Harlow and S. L. Phoenix, Adv. Appl. Probab. 14, 68 (1982).

[32] R. L. Smith, Proc. R. Soc. London Ser. A 382, 179 (1982).

[33] R. C. Hidalgo, Y. Moreno, F. Kun, and H. J. Herrmann, Phys. Rev. E 65, 046148 (2002).

[34] S. Pradhan, P. Bhattacharyya, and B. K. Chakrabarti, Phys. Rev. E 66, 016116 (2002).

[35] S. Roy and P. Ray, Europhys. Lett. 112, 26004 (2015).

[36] P. Ray and B. K. Chakrabarti, Solid State Commun. 53, 477 (1985).

[37] C. Roy, S. Kundu, and S. S. Manna, Phys. Rev. E 87, 062137 (2013).

[38] S. Roy, Phys. Rev. E 96, 042142 (2017).

[39] S. J. Kim, W. T. Jung, Y. S. Kong, and W. G. Kim, in Proceedings of the 2nd ECCC Creep Conference, Zurich, 2009, edited by I. A. Shilbi and S. R. Holdsworth (DEStect, Lancaster, 2009).

[40] M. Evans, J. Mater. Process. Technol. 54, 171 (1995).

[41] M. Evans and A. R. Ward, Mater. Sci. Technol. 16, 1149 (2000).

[42] M. Evans, Materials 10, 1190 (2017).

[43] S.-J. Kim, Y.-S. Kong, Y.-J. Roh, and W.-G. Kim, Mat. Sci. Eng. A 483-484, 529 (2008). 